\title{
Concept Implicate Tree for Description Logics
}

\author{
Tingting Zou* and Ansheng Deng \\ Information Science and Technology College, Dalian Maritime University, Dalian, 116026, China
}

\begin{abstract}
Description logics is a class of knowledge representation languages with high expressive power, and the computational complexities of the queries of these expressive description logics are defined as PSPACE-complete. Moreover, knowledge compilation can be regarded as a new direction of research for dealing with the computational intractable reasoning problems. In fact, knowledge compilation based on description logic has been investigated in recent years. However, when the compiled knowledge base is exponential as compared to original knowledge base, the queries are not. Therefore, we proposed a new knowledge compilation method for description logic to solve the queries in linear time depending on the size of the query. In this paper, we first introduced the concept implicate tree for the ALC concept. Then, we present an algorithm, which can transform an ALC concept into an equivalent concept implicate tree, and proved that each branch of the tree is an implicate of this concept. Finally, we proved that the queries are computable in linear time. The proposed method has an important property that no matter how large the concept implicate tree is, any query can be resolved in linear time depending on the size of the query.
\end{abstract}

Keywords: ALC, Description logic, Knowledge compilation, PSAPCE-complete, Algorithm Build CIT, Tractable querying.

\section{INTRODUCTION}

Description logics (DL) is a class of knowledge representation languages, which can model an application domain of interest by a structured and formally well-understood method[1]. In fact, DLs can be used in various areas, for example, Semantic Web [2, 3], Ontologies [4], and software engineering [5]. Schmidt-Schauß and Smolka proposed description logic ALC, and proved that the queries of ALC concepts were PSPACE-complete [6]. Subsequently, Donini et al. stated that the queries of ALCN concepts were also PSPACE-complete [7]. With the rapid development of DLs, abundant DL systems have been presented, such as SHIN [8], SHIQ [9], SHOIQ [10, 11], SROIQ [12] and so on. However, the computational complexities of the queries of these expressive description logics are intracte.

Knowledge compilation has emerged as a new direction of research for dealing with the computational intractability of general propositional reasoning [13]. In this approach, reasoning process is split into two phases: off-line compilation and on-line query-answering [14]. In the first phase, the propositional knowledge base is compiled into some target language, which is typically tractable. In the latter phase, the query is actually answered by using the compiled knowledge base of the first phase. The key of this approach is that knowledge compilation needs to be done only once to be accessible for different queries. Hence, the compiling time can be amortized by many queries concerning the compiled knowledge base [15]. There are many target languages for knowledge compilation, such as prime implicate [16], DNNF

\footnotetext{
*Address correspondence to this author at the Information Science and Technology College, Dalian Maritime University, Dalian, Liaoning, 116026, China; Tel/Fax: 041184723122; E-mail: zoutt@dlmu.edu.cn
}

[17], and so on. In fact, the queries for these target languages are based on polynomial time or linear time dependent on the size of the compiled knowledge base. Moreover, Murray and Rosenthal introduced the reduced implicate tree that is a target language for knowledge compilation, and proved that a query can be done in linear time considering the size of the query [18-20].

As mentioned above, knowledge compilation is an efficient method to deal with intractable problems. Therefore, many researchers have conducted their studies on knowledge compilation for description logics in recent years. Selman and Kautz compiled a concept of DL FL into two approximate concepts of $\mathrm{DL} \mathrm{FL}^{-}$, being the first knowledge compilation method for DL [21]. Subsequently, Furbach and Obermajer introduced the linkless concept description for ALC concepts, which can be regarded as a target language for knowledge compilation, by presenting an algorithm that transformed ALC concept to equivalent linkless concept description, and proved that queries for such descriptions were resolved in linear time based on the size of the descriptions [22]. Later, they used this technique for precompiled ALC concepts and TBoxes so that queries can be addressed in linear time [23, 24]. Moreover, Bienvenu proposed the prime implicate normal form for ALC concepts, and concluded that the queries of such forms are based on polynomial time [25]. Tingting Zou et al., proposed a novel knowledge compilation method for description logic based on the concept extension rule [26].

In fact, the queries of these methods were also based on the polynomial time or linear time depending on the size of the compiled knowledge base. However, when the compiled knowledge base was exponential in terms of the size of the original knowledge base, the queries were not addressed rapidly. This paper aims to further improve the reduced implicate tree for propositional logic, to make it a much more 
efficient description logic for target language. Therefore, we proposed a new knowledge compilation method for the description logic based on the concept implicate tree, for which the queries can be addressed in linear time based on the size of the query regardless of the size of the compiled knowledge base.

In this paper, we first introduced the concept implicate tree for ALC concept, which is a target language for knowledge compilation, and defined the concept represented by concept implicate tree. Then, an algorithm was presented, which can transform ALC concepts into the concept implicate trees. Moreover, we proved that the concept represented by this concept implicate tree was equivalent to the original ALC concept, and each branch of the tree was an implicate of the original concept. Furthermore, we explained that the satisfiability-testing and tautology-testing were carried out in linear time with respect to the concept implicate tree. Finally, we presented an algorithm determining the subsumption of two concepts, and proved that subsumption-testing was computable in linear time based on the size of the query. In a word, this method has an important property that no matter how large the concept implicate tree is, any query can be assessed in linear time depending on the size of the query.

The rest of this paper is organized as follows. In section 2 , the concept implicate tree is defined. Section 3 presents the process of transforming an ALC concept into an equivalent concept implicate tree. In Section 4, it is proved that the queries are computable in linear time. Section 5 summarizes the main results.

\section{CONCEPT IMPLICATE TREE}

Let $C_{A}, R_{A}$ and $I_{A}$ be the pairwise disjointing sets of atomic concepts, abstract role names, and abstract individuals, respectively, and $\sqcup$ operation be the concept disjunction, with $\sqcap$ operation being the concept conjunction.

Definition 1. Literal L, ALC concept C, and clausal concept cl, are defined as follows:

$$
\begin{aligned}
L & :=|\perp| A|\neg A| \exists R . L \mid \forall R . L, \\
C & :=L|C \sqcup C| C \sqcap C, \\
c l & :=L \mid c l \sqcup c l,
\end{aligned}
$$

where $A \in C_{A}, R \in R_{A}$.

Definition 2. In literal $L, A$ or $\neg A$ is called the concept literal, and $A$ is known as the atomic concept variable, with the form $J R . L$ or $\nabla R . L$ known as the role concept literal and also as the role concept variable.

For any concept $C, V_{C o n}(C)$ denotes the set of all atomic concept variables of $C$, and $V_{R o l}(C)$ denotes the set of all role concept variables of $C$. Moreover, depth $(Q R . L)$ denotes the number of the form $Q R$ in $Q R . L, Q \in\{\forall, \exists\}$. For example, if

$$
C=\left(A_{1} \sqcup \neg A_{2} \sqcup \exists R_{1} \cdot \neg A_{3}\right) \sqcap\left(A_{1} \sqcup \forall R_{1} \cdot \exists R_{2} . A_{2}\right),
$$

then,

$$
\begin{aligned}
& C_{A}=\left\{A_{1}, A_{2}, A_{3}\right\}, R_{A}=\left\{R_{1}, R_{2}\right\}, \\
& V_{\text {Con }}(C)=\left\{A_{1}, A_{2}\right\}, V_{R o l}(C)=\left\{\exists R_{1} \cdot \neg A_{3}, \forall R_{1} \cdot \exists R_{2} \cdot A_{2}\right\}, \\
& \operatorname{depth}\left(\exists R_{1} \cdot \neg A_{3}\right)=1, \operatorname{depth}\left(\forall R_{1} \exists R_{2} \cdot A_{2}\right)=2 .
\end{aligned}
$$

Let $C_{1}$, and $C_{2}$ be the ALC concepts, and $B$ is the subconcept of $C_{1} . C_{1}\left[C_{2} / B\right]$ is used to refer to the new concept, which is produced by substituting $C_{2}$ for every occurrence of $B$ that is not in the scope of role restriction in $C_{1}$. Especially, if $C_{2}$ is $T$ or I, $B$ is an atomic concept variable $A$ or role concept variable $Q R . L, Q \in\{\forall, \exists\}$, then $C_{1}[T / B]$ denotes that $\mathrm{T}$ is substituted for $B$, and $\mathrm{I}$ for $\neg B$, but $\mathrm{T}$ or $\mathrm{I}$ is not substituted for $Q R . B$ or $Q R . \neg B$.

Definition 3. Reduction rules are defined as follows:

$$
\begin{array}{ll}
C[B / B \sqcup \perp], & C[\perp / B \sqcap \perp], \\
C[B / B \sqcap \top], & C[\top / B \sqcup \top], \\
C[\perp / B \sqcap \neg B], & C[\top / B \sqcup \neg B], \\
C[\perp / \exists R . \perp], & C[\top / \forall R . \top] .
\end{array}
$$

Definition 4. Let $V_{C o n}(C)-\left\{A_{-}, A_{2}, \ldots, A_{n}\right\}$ be the set of atomic concept variables of ALC concept $C$, and

$$
V_{R o l}(C)=\left\{Q R_{i} \cdot L_{j} \mid Q \in\{\exists, \forall\}, 1 \leq i \leq p, 1 \leq j \leq q\right\}
$$

be the set of role concept variables of ALC concept $C$. A partial ordering relation $\prec$ on sets $V_{C o n}(C)$ and $V_{R o l}(C)$ is defined as follows:

(1) $A \prec Q R . L$ iff $A \in V_{\text {Con }}(C), Q R . L \in V_{R o l}(C)$;

(2) $A_{i} \prec A_{j}$ iff $\mathrm{i}<\mathrm{j}$;

(3) $Q R_{i} \cdot L_{j} \prec Q R_{r} \cdot L_{s}$ iff $\operatorname{depth}\left(Q R_{i} \cdot L_{j}\right)<\operatorname{depth}\left(Q R_{r} \cdot L_{s}\right)$;

(4) $Q R_{i} . L \prec Q R_{r} . L$ iff $\mathrm{i}<\mathrm{r}$;

(5) $Q R . L_{j} \prec Q R . L_{s}$ iff $\mathrm{j}<\mathrm{s}$;

(6) $\exists R . L \prec \forall R . L$.

In this paper, we assumed that $V_{C o n}(C)$ and $V_{\text {Role }}(C)$ satisfy this partial ordering relation, that is to say, $V_{C o n}(C)$ and $V_{\text {Role }}(C)$ are the ordered sets. For simplicity, we write $V_{C o n}(C)$ as $V_{\text {Con }}$, and $V_{\text {Role }}(C)$ as $V_{\text {Role }}$.

Definition 5. Let $C$ be an ALC concept, and $c l$ be a clausal concept. Then $c l$ is an implicate of $C$ if and only if $\mathrm{C}-\mathrm{Cl}$. Moreover, $\mathrm{cl}$ is a prime implicate of $C$ if and only if $C-\mathrm{Cl}$, and there does not exist an implicate $c l^{\prime}$ of $C$ such that $C \sqsubseteq c l^{\prime} \sqsubseteq c l$ and $c l \sqsubseteq c l^{\prime}$.

Definition 6. Concept implicate tree (CIT) $T$ for ALC concept $C$ is a tree defined as follows:

(1) If $C$ is tautology, then $T$ contains only one root node labelled as $\mathrm{T}$; 
(2) If $C$ is unsatisfiable, then $T$ contains only one root node labeled as I;

(3) Otherwise, root node of $T$ is labelled as $I$, and for any implicate $c l=L_{1} \sqcup L_{2} \sqcup \cdots \sqcup L_{m}$ of $\mathrm{C}$, root node has a child node labelled as $L_{1}$, which is the root of a subtree containing a branch with labels corresponding to $L_{2} \sqcup \cdots \sqcup L_{m}$.

(4) $T$ is reduced by using the rules in Definition 3, until no rule can be applied.

According to definition 6 , it can be observed that each branch of CIT $T$ is an implicate of $C$.

Definition 7. Let $T$ be the concept implicate tree for ALC concept $C$. Then concept $C_{T}$ that is represented by the tree $T$ is defined as follows:

(1) If $T$ has only one node, then $C_{T}$ is the label of this node.

(2) Otherwise, $C_{T}$ is the concept disjunction of two concepts; one concept is the label of the root, and the other is the concept conjunction of labels of all branches of this root.

Example 1: ALC concept

$$
\begin{gathered}
C=\left(A_{1} \sqcup A_{2} \sqcup \forall R_{2} . \neg A_{4}\right) \sqcap\left(A_{1} \sqcup \forall R_{1} \cdot \exists R_{2} . A_{5}\right) \\
\sqcap\left(\neg A_{1} \sqcup \neg A_{2} \sqcup \forall R_{1} . \exists R_{2} . A_{5}\right)
\end{gathered} .
$$

where, $V_{C}(C)=\left\{A_{1}, A_{2}\right\}, V_{R}(C)=\left\{\forall R_{2} . \neg A_{4}, \forall R_{1} . \exists R_{2} . A_{5}\right\}$.

Then, the concept implicate tree $T$ of $C$ is shown as follows, and each branch of $T$ is an implicate of $C$. For example,

$$
\begin{aligned}
& A_{1} \sqcup A_{2} \sqcup \forall R_{2} . \neg A_{4}, A_{1} \sqcup A_{2} \sqcup \exists R_{2} . A_{4} \sqcup \forall R_{1} . \exists R_{2} . A_{5}, \\
& \text { and } \neg A_{1} \sqcup \neg A_{2} \sqcup \forall R_{1} . \exists R_{2} . A_{5},
\end{aligned}
$$

are all implicates of $C$.

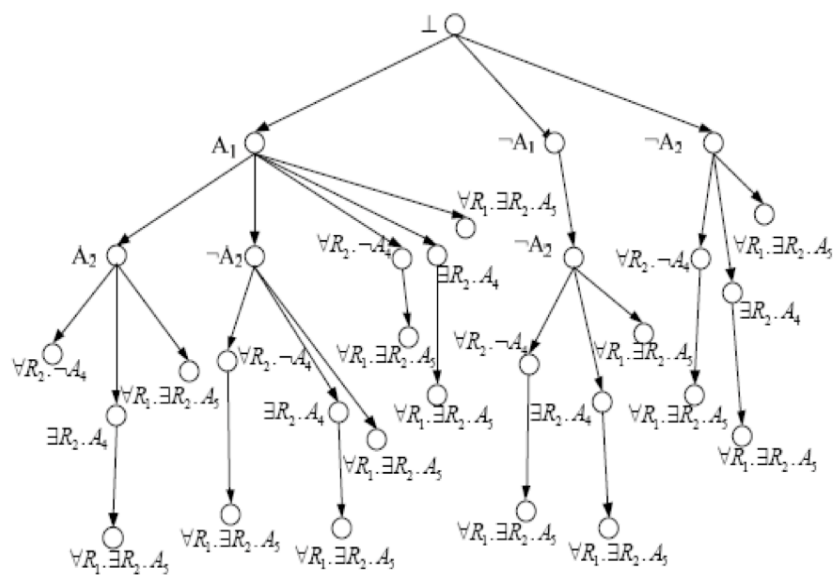

Moreover, the concept $C_{T}$ is

$$
\begin{aligned}
& C_{T}=\left(A _ { 1 } \sqcup \left(\left(A _ { 2 } \sqcup \left(\forall R_{2} \cdot \neg A_{4} \sqcap\left(\exists R_{2} \cdot A_{4} \sqcup \forall R_{1} \cdot \exists R_{2} \cdot A_{5}\right)\right.\right.\right.\right. \\
& \left.\left.\sqcap \forall R_{1} \cdot \exists R_{2} \cdot A_{5}\right)\right) \sqcap\left(\neg A _ { 2 } \sqcup \left(\left(\forall R_{2} \cdot \neg A_{4} \sqcup \forall R_{1} \cdot \exists R_{2} \cdot A_{5}\right)\right.\right. \\
& \left.\left.\sqcap\left(\exists R_{2} \cdot A_{4} \sqcup \forall R_{1} \cdot \exists R_{2} \cdot A_{5}\right) \sqcap \forall R_{1} \cdot \exists R_{2} \cdot A_{5}\right)\right) \\
& \sqcap\left(\forall R_{2} \cdot \neg A_{4} \sqcup \forall R_{1} \cdot \exists R_{2} \cdot A_{5}\right) \sqcap\left(\exists R_{2} \cdot A_{4} \sqcup \forall R_{1} \cdot \exists R_{2} \cdot A_{5}\right) \\
& \left.\left.\sqcap \forall R_{1} \cdot \exists R_{2} \cdot A_{5}\right)\right) \sqcap\left(\neg A _ { 1 } \sqcup \left(\neg A _ { 2 } \sqcup \left(\left(\forall R_{2} \cdot \neg A_{4} \sqcup \forall R_{1} \cdot \exists R_{2} \cdot A_{5}\right)\right.\right.\right. \\
& \left.\left.\left.\sqcap\left(\exists R_{2} \cdot A_{4} \sqcup \forall R_{1} \cdot \exists R_{2} \cdot A_{5}\right) \sqcap \forall R_{1} \cdot \exists R_{2} \cdot A_{5}\right)\right)\right) \\
& \sqcap\left(\neg A _ { 2 } \sqcup \left(\neg A _ { 2 } \sqcup \left(\left(\forall R_{2} \cdot \neg A_{4} \sqcup \forall R_{1} \cdot \exists R_{2} \cdot A_{5}\right)\right.\right.\right. \\
& \left.\left.\sqcap\left(\exists R_{2} \cdot A_{4} \sqcup \forall R_{1} \cdot \exists R_{2} \cdot A_{5}\right) \sqcap \forall R_{1} \cdot \exists R_{2} \cdot A_{5}\right)\right)
\end{aligned}
$$

\section{TRANSFORMATION}

In this section, we introduced a method to transform an ALC concept into an equivalent concept implicate tree, and proved that each branch of the tree is an implicate of this concept. Let $\operatorname{Cimp}(C)$ be the sets of implicates of concept $C$.

Theorem 1. Let $\mathrm{C}$ be the ALC concept, $\mathrm{V}_{\mathrm{Con}}$ be the atomic concept variables of $\mathrm{C}, \mathrm{V}_{\mathrm{Rol}}$ be a role concept variables of $\mathrm{C}$, and clausal concept $\mathrm{cl}$ be an implicate of $\mathrm{C}$. If there exists an atomic concept variable A (or a role concept variable $Q R . L, \quad Q \in\{\forall, \exists\}), \quad$ such $\quad$ that $A \in V_{C o n}(C)$ and $A \notin V_{C o n}(c l)$ (or $\left.Q R . L \in V_{R o l}(C), Q R . L \notin V_{R o l}(c l)\right)$, then

$$
\begin{aligned}
& c l \in \operatorname{Cimp}(C[\perp / A]) \cap \operatorname{Cimp}(C[\top / A]) \\
& (\text { or } c l \in \operatorname{Cimp}(C[\perp / Q R . L]) \cap \operatorname{Cimp}(C[\top / Q R . L])) .
\end{aligned}
$$

Proof. (1) We first proved that $c l \in \operatorname{Cimp}(C[\top / A])$. Let $I=\left\langle\Delta^{I}, \bullet^{I}\right\rangle$ be a model of concept $C[\top / A]$, therefore, $(C[\top / A])^{I} \neq \varnothing$.Following this , we extended $I$ to $I^{\prime}=<\Delta^{I^{\prime}}, \bullet^{I^{\prime}}>$ by setting $\Delta^{I}=\Delta^{I^{\prime}}, \quad A^{I^{\prime}}=\Delta^{I}$, then $C^{I^{\prime}}=(C[\top / A])^{I} \neq \varnothing$. Therefore, $I^{\prime}$ became the model of $C$. Because clausal concept $\mathrm{cl}$ was an implicate of $C$, therefore, $C^{I^{\prime}} \mid c l^{I^{\prime}}$. Since $A \notin V_{C o n}(c l)$, then $c l^{I^{\prime}}=c l^{I}$. Hence, $(C[\top / A])^{I}=C^{I^{\prime}} \subseteq c l^{I^{\prime}}=c l^{I}$. Thus, $C[\top / A] \sqsubseteq c l$. According to the Definition 5, $c l \in \operatorname{Cimp}(C[\top / A])$. The proof for $C[\top / Q R . L]$ is similar.

(2)Following this, we proved that $c l \in \operatorname{CImp}(C[\perp / A])$. Let $I=<\Delta^{I},\left\langle^{I}>\right.$ be the model of concept $C[\perp / A]$, therefore,$(C[\perp / A])^{I} \neq \varnothing$. Now, we extended $I$ to $\left.I^{\prime}=<\Delta^{I^{\prime}}, \bullet^{I^{\prime}}\right\rangle$ by setting $\Delta^{I}=\Delta^{I^{\prime}}, \quad A^{I^{\prime}}=\rightarrow$, then $C^{I^{\prime}}=(C[\perp / A])^{I} \neq \varnothing$. Therefore, $\quad I^{\prime}=<\Delta^{I}, \bullet^{I^{\prime}}>$ is the model of concept $C$. Because clausal concept $c l$ was an implicate of concept $C$, therefore, $C^{I^{\prime}} \subseteq c l^{I^{\prime}}$. Since $A \notin V_{C o n}(c l), \quad$ then $\quad c l^{I^{\prime}}=c l^{I}$. Hence, $(C[\perp / A])^{I}=C^{I^{\prime}} \subseteq c l^{I^{\prime}}=c l^{I}$. Thus, $C[\perp / A] \sqsubseteq c l$. According to the Definition 5, $c l \perp \operatorname{CImp}(C[\perp / A])$. The proof for $C[\perp / Q R . L]$ is similar. 
Above all, $\quad c l \in \operatorname{Cimp}(C[\perp / A]) \cap \operatorname{Cimp}(C[\top / A]) \quad$ or $c l \in \operatorname{Cimp}(C[\perp / Q R . L]) \cap \operatorname{Cimp}(C[\top / Q R . L])$.

Theorem 2. Let $C_{1}$ and $C_{2}$ be the ALC concepts. Then, $\operatorname{Cimp}\left(C_{1} \sqcup C_{2}\right)=\operatorname{Cimp}\left(C_{1}\right) \cap \operatorname{Cimp}\left(C_{2}\right)$.

Proof. $\quad(\Rightarrow)$ Assuming that a clausal concept $c l \in \operatorname{Cimp}\left(C_{1} \sqcup C_{2}\right), \quad$ we proved that $c l \in \operatorname{Cimp}\left(C_{1}\right) \cap \operatorname{Cimp}\left(C_{2}\right)$. (1) To see that $c l \in \operatorname{Cimp}\left(C_{1}\right)$. Let $I=\left\langle\Delta^{I}, \bullet^{I}\right\rangle$ be the model of $C_{1}$, then $I$ is also a model of $\quad C_{1} \sqcup C_{2}$. Since $\quad c l \in \operatorname{Cimp}\left(C_{1} \sqcup C_{2}\right)$, then $\left(C_{1} \sqcup C_{2}\right)^{I}=C_{1}^{I} \cup C_{2}^{I} \subseteq c l^{I}$. Therefore, $C_{1}^{I} \subseteq c l^{I}, C_{1} \sqsubseteq c l$. Thus $c l \in \operatorname{Cimp}\left(C_{1}\right)$. (2) To see that $c l \in \operatorname{CImp}\left(C_{2}\right)$. Let $I=\left\langle\Delta^{I}, \bullet^{I}\right\rangle$ be the model of concept $C_{2}$, then $I$ is also a model of $C_{1} \sqcup C_{2}$. Since $c l \in \operatorname{Cimp}\left(C_{1} \sqcup C_{2}\right)$, then $\left(C_{1} \sqcup C_{2}\right)^{I}=C_{1}^{I} \cup C_{2}^{I} \subseteq c l^{I}$. therefore, $C_{2}^{I} \subseteq c l^{I}, C_{2} \supsetneqq c l$. Thus $c l \in \operatorname{Cimp}\left(C_{2}\right)$. Therefore, $c l \in \operatorname{Cimp}\left(C_{1}\right) \cap \operatorname{Cimp}\left(C_{2}\right)$, and $\operatorname{Cimp}\left(C_{1} \sqcup C_{2}\right) \subseteq \operatorname{Cimp}\left(C_{1}\right) \cap \operatorname{Cimp}\left(C_{2}\right)$.

$(\Leftarrow) \quad$ Assuming that $c l \in \operatorname{Cimp}\left(C_{1}\right) \cap \operatorname{Cimp}\left(C_{2}\right)$, we proved that $c l \in \operatorname{Cimp}\left(C_{1} \sqcup C_{2}\right)$. Let $I=\left\langle\Delta^{I}, \bullet^{I}\right\rangle$ be the model of $C_{1} \sqcup C_{2}$, then $I$ is a model of $C_{1}$ or $C_{2}$. There are three cases:

(1) $I$ is a model of $C_{1}$, but not a model of $C_{2}$. Since $c l \in \operatorname{Cimp}\left(C_{1}\right), \quad$ then $\quad C_{1}^{I} \subseteq c l^{I}$. Therefore, $\left(C_{1} \sqcup C_{2}\right)^{I}=C_{1}^{I} \cup C_{2}^{I}=C_{1}^{I} \cup \varnothing=C_{1}^{I} \subseteq c l^{I}$.

(2) $I$ is a model of $C_{2}$, but not a model of $C_{1}$. Since $c l \in \operatorname{Cimp}\left(C_{2}\right)$, then $C_{2}^{I} \mid c l^{I}$.Therefore,

$$
\left(C_{1} \sqcup C_{2}\right)^{I}=C_{1}^{I} \cup C_{2}^{I}=\varnothing \cup C_{2}^{I}=C_{2}^{I} \subseteq c l^{I} .
$$

(3) $I$ is a model of $C_{1}$, and also is a model of $C_{2}$. Since $c l \in \operatorname{Cimp}\left(C_{1}\right) \cap \operatorname{Cimp}\left(C_{2}\right)$, then $C_{1}^{I} \subseteq c l^{I}, C_{2}^{I} \subseteq c l^{I}$. Therefore, $\left(C_{1} \sqcup C_{2}\right)^{I}=C_{1}^{I} \cup C_{2}^{I} \subseteq c l^{I}$. Therefore,

$$
\mathrm{cl} \in \operatorname{Cimp}\left(C_{1} \sqcup C_{2}\right) \text {, and }
$$

$$
\operatorname{Cimp}\left(C_{1}\right) \cap \operatorname{Cimp}\left(C_{2}\right) \subseteq \operatorname{Cimp}\left(C_{1} \sqcup C_{2}\right)
$$

Above all, $\operatorname{Cimp}\left(C_{1} \sqcup C_{2}\right)=\operatorname{Cimp}\left(C_{1}\right) \cap \operatorname{Cimp}\left(C_{2}\right)$.

Theorem 3. Let $c l$ be a clausal concept without the concept variable $E(E=A$ or $E=Q R . L)$, and $C$ be any ALC concept. Then $\mathrm{cl}$ is an implicate of $C$ if $c l$ is an implicate of $C[\perp / E] \sqcup C[\top / E]$.

Proof. According to Theorem 1 and Theorem 2, it is obvious that this conclusion is correct.

According to Theorem 3, the set of implicates of $C$ consists of three parts: (1) The first part is the set of implicates concept variables $E, E=A$ or $E=Q R . L$; (2) The second part is the set of implicate concept variable $\neg E, E=A$ or $E=Q R . L$ QR.L; (3) the third part is the set of implicates of $C[\perp / E] \sqcup C[\top / E]$, which neither contains concept variable E nor the concept variable $\neg E, E=A$ or $E=Q R . L$.

Therefore, a concept implicate tree $T$ can be regarded as a ternary tree, with each node having three subtrees except the leaf node. The first subtree is $T_{1}$, the second subtree is $T_{2}$, and the third subtree is $T_{3}$. Let $N$ be a node labelling $E_{i}$, and $T_{1}, T_{2}, T_{3}$ be the three subtrees of node $N$. Then the root node of $T_{1}$ is labelled as $E_{i+1}$, and $T_{1}$ contains the sets of implicates occurring $E_{i+1}$. Moreover, the root node of $T_{2}$ is labelled as $\neg E_{i+1}$, and $T_{2}$ contains the sets of implicates occurring $\neg E_{i+1}$. Furthermore, the root node of $T_{3}$ is labelled as $\perp$ , and $T_{3}$ contains the set of implicates not occurring $E_{i+1}$ and $\neg E_{i+1}$, which are the intersection of $T_{1}$ and $T_{2}$ irrespective of $E_{i+1}$ and $\neg E_{i+1}$.

Therefore, a method was proposed to build a concept implicate tree of a given concept. First, the structure of a node of the tree was defined as shown in Fig. (1), then the algorithms Simplify and BuildCIT were presented as shown in Figs. (2) and (3). Algorithm BuildCIT has four input parameters,

$$
\begin{aligned}
& \text { Structure CITnode(label: string, } \\
& \text { leaf: boolean, } \\
& \text { first: } \uparrow \text { CITnode, } \\
& \text { second: } \uparrow \text { CITnode, } \\
& \text { third: } \uparrow \text { CITnode); }
\end{aligned}
$$

Fig. (1). Structure CITnode.

\section{Algorithm Simplify}

Input: concept $C$

Output: simplified concept of $C$.

1. Applying the following rules until no rule can be applied:

$$
\begin{aligned}
& C=C[\perp / F \sqcap \perp], C=C[F / F \sqcup \perp], \\
& C=C[F / F \sqcap \top], C=C[\top / F \sqcup \top], \\
& C=C[\perp / F \sqcap \neg F], C=C[\top / F \sqcup \neg F], \\
& C=C[\perp / \exists R . \perp], C=C[\top / \forall R . \top] .
\end{aligned}
$$

2. Return $C$.

Fig. (2). Algorithm simplify.

Where, ALC is a concept $C$, with the set of atomic concept variables $V_{C o n}$, the set of role concept variables $V_{\text {Rol }}$, node $N$, one output parameter, and the concept implicate tree $T$. Initially, $V_{C o n}=V_{C o n}(C), V_{R o l}=V_{R o l}(C), N=$ nil. For every node, the algorithm BuildCIT first built the first subtree and the second subtree, followed by the third subtree based on 
computing the intersection of the first two subtrees which is illustrated in Fig. (4).

Example 2. For the concept $C$ in example 1, the algorithm BuildCIT( $C, V_{C o n}, V_{R o l}$, nil) built a tree $T$ as follows:

First, a new CITnode $N^{\prime}$ was built, which is root node of tree $T$, and $N^{\prime}$.label $=\perp$, returning to $\operatorname{BuildCIT}\left(C, V_{C o n}\right.$, $\left.V_{R o l}, N^{\prime}\right)$.

For BuildCIT( $\left.C, V_{C o n}, V_{R o l}, N^{\prime}\right)$ :

1) Let $N=N^{\prime}$;

2) An atomic concept variable $A$ was selected w $_{1}$;

3) $C_{1}=\operatorname{Simplify}(C[\perp / E])=\left(A_{2} \sqcup \forall R_{2} \cdot \neg A_{4}\right) \sqcap \forall R_{1} \cdot \exists R_{2} \cdot A_{5}$ $C_{2}=\operatorname{Simplify}(C[\top / E])=\neg A_{2} \sqcup \forall R_{1} \cdot \exists R_{2} \cdot A_{5}$;

4) A new CIT node $N_{1}$ of tree $T$ was built with $N_{1}$.label= $A_{1}, N$.first $=N_{1}$, call BuildCIT $\left(C_{1},\left\{A_{2}\right\}, V_{R o l}, N_{1}\right)$;

\section{Algorithm BuildCIT}

Input: concept $C, V_{C o n}, V_{R o l}$, node $N$;

Output: concept implicate tree $T$;

1. If $C=\perp$ or $C=\top$, then

build a new CITnode $N^{\prime}$ of tree $T$, and $N^{\prime}$.label $=C$, returen $T$.

2. If $N=$ nil, then

build a new CITnode $N^{\prime}$, which is root node of tree $T$, and $N^{\prime}$.label $=\perp$, return BuildCIT $\left(C, V_{C o n}, V_{R o l}, N^{\prime}\right)$.

3. If $V_{C o n}=\varnothing$, then

select the first role concept variable

$E=Q R_{j} \cdot L_{i} \in V_{R o l}$;

Else, select the first atomic concept variable $E=A \in V_{C o n}$.

4. Let $C_{1}=\operatorname{Simplify}(C[\perp / E])$,

$C_{2}=\operatorname{Simplify}(C[\top / E])$.

5. If $C_{1}=\perp$, then

build a new CITnode $N_{1}$ of tree $T$, and $N_{1}$.label $=E$, $N_{1}$.leaf $=$ True, $N$.first $=N_{1}$.

6. If $C_{2}=\perp$, then

build a new CITnode $N_{2}$ of tree $T$, and $N_{2}$.label $=\neg E$ $N_{2}$.leaf $=$ True,

$N$.second $=N_{2}$.

7. If $C_{1}=\top$, then $N$.first $=$ nil, $N$.third $=$ nil;

Else

build a new CITnode $N_{1}$ of tree $T$, and $N_{1}$.label $=E$, $N$.first $=N_{1}$,

if $V_{C o n} \neq \varnothing$, then

call BuildCIT $\left(C_{1}, V_{C o n^{-}}\{E\}, V_{R o l}, N_{1}\right)$;

else

call BuildCIT $\left(C_{1}, V_{\mathrm{Con}}, V_{\mathrm{Rol}}-\{E\}, N_{1}\right)$.
8. If $C_{2}=\top$, then $N$.second=nil, $N$.third=nil;

Else

build a new CITnode $N_{2}$ of tree $T$, and $N_{2}$.label $=\neg E$, $N$.second $=N_{2}$,

if $V_{C o n} \neq \varnothing$, then

call BuildCIT $\left(C_{2}, V_{C^{\prime} n^{-}}\{E\}, V_{R o l}, N_{2}\right)$;

else

call BuildCIT $\left(C_{2}, V_{C o n}, V_{R o l}-\{E\}, N_{2}\right)$.

9. If $\left(N_{1}\right.$.leaf and $N_{2}$.leaf $)$, then

delete node $N_{1}, N_{2}$, and $N$.leaf=True, return $T$.

10. If ( $N$.first $\neq$ nil and $N$.second $\neq$ nil), then

build a new CITnode $N_{3}$ of tree $T$, and $N$.third $=N_{3}$, call BuildThird( $N$.first, $N$.second, $N_{3}$ ),

and $N_{3}$.label $=\perp$.

11. Return $T$.

Fig. (3). Algorithm BuildCIT.

Algorithm BuildThird

Input: CIT nodes $N_{1}, N_{2}, N_{3}$;

Output: tree $T$.

1. $\quad N_{3}$.label $=N_{1}$.label.

2. If $N_{1}$.leaf $==$ true and $N_{2}$.leaf $==$ true, then $N_{3}$.leaf $=$ true, return $T$.

3. If $N_{1}$.leaf $==$ true,

then $N_{3}$.first $=N_{2}$.first, $N_{3}$. second $=N_{2}$. second,

$N_{3}$.third $=N_{2}$.third, return $T$.

4. If $N_{2}$.leaf $==$ true,

then $N_{3}$.first $=N_{1}$. first, $N_{3}$. second $=N_{1}$. second,

$N_{3}$.third $=N_{1}$.third, return $T$.

5. If $N_{1}$.first=nil or $N_{2}$.first=nil, then $N_{3}$.first=nil;

Else

build a new CITnode $N_{31}$ of tree $T$,

$N_{3}$. first $=N_{31}$,

call BuildThird $\left(N_{1}\right.$.first, $N_{2}$.first, $\left.N_{31}\right)$.

6. If $N_{1}$.second $=$ nil or $N_{2}$.second $=$ nil,

then $N_{3}$.second =nil;

Else

build a new CITnode $N_{32}$ of tree $T$,

$N_{3}$.second $=N_{32}$,

call BuildThird $\left(N_{1}\right.$.second, $N_{2}$.second, $\left.\mathbf{N}_{32}\right)$.

7. If $N_{1}$.third $=$ nil or $N_{2}$.third $=$ nil,

then $N_{3}$.third =nil;

Else

build a new CITnode $N_{33}$ of tree $T$,

$N_{3} \cdot$ third $=N_{33}$,

call BuildThird $\left(N_{1}\right.$.third, $N_{2}$.third, $\left.N_{33}\right)$.

8. $\quad$ Return $T$.

Fig. (4). Algorithm BuildThird. 
5) A new CIT node $N_{2}$ of tree $T$ was built with $N_{2}$.label $=-$ $A_{1}, N$.second $=N_{2}$, call BuildCIT $\left(C_{2},\left\{A_{2}\right\}, V_{R o l}, N_{2}\right)$;

6) A new CITnode $N_{3}$ of tree $T$ was built with $N$.third $=N_{3}$, call BuildThird $\left(N_{1}, N_{2}, N_{3}\right), N_{3}$.label $=\perp$;

7) Returning to $T$.

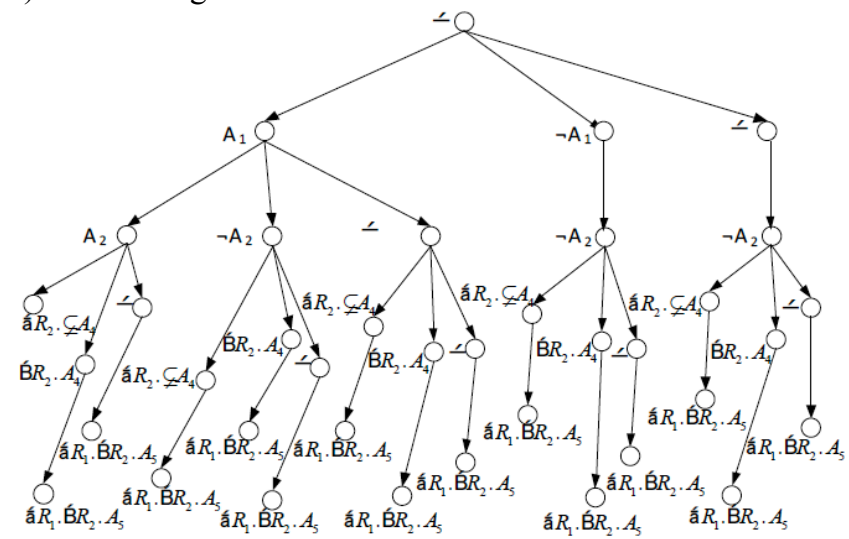

In this algorithm, three algorithms are addressed, BuildCIT $\left(\mathrm{C}_{1},\left\{\mathrm{~A}_{2}\right\}, \mathrm{V}_{\text {Rol }}, \mathrm{N}_{1}\right), \quad$ BuildCIT $\left(\mathrm{C}_{2}, \quad\left\{\mathrm{~A}_{2}\right\}, \mathrm{V}_{\mathrm{Rol}}, \mathrm{N}_{2}\right)$, and BuildThird $\left(\mathrm{N}_{1}, \mathrm{~N}_{2}, \mathrm{~N}_{3}\right)$. The algorithms BuildCIT $\left(\mathrm{C}_{1}\right.$, $\left.\left\{\mathrm{A}_{2}\right\}, \mathrm{V}_{\mathrm{Rol}}, \mathrm{N}_{1}\right)$ and BuildCIT $\left(\mathrm{C}_{2},\left\{\mathrm{~A}_{2}\right\}, \mathrm{V}_{\mathrm{Rol}}, \mathrm{N}_{2}\right)$ iterate the process of algorithm BuildCIT(C, $\left.\mathrm{V}_{\mathrm{Con}}, \mathrm{V}_{\mathrm{Rol}}, N^{\prime}\right)$, and build the first and second subtrees of node $N^{\prime}$. Algorithm BuildThird $\left(\mathrm{N}_{1}, \mathrm{~N}_{2}, \mathrm{~N}_{3}\right)$ builds the third subtree of node $N^{\prime}$. Finally, algorithm BuildCIT(C, $\left.\mathrm{V}_{\mathrm{Con}}, \mathrm{V}_{\mathrm{Rol}}, N^{\prime}\right)$ returns the concept implicate tree $T$ of $C$ as shown below.

Theorem 4. Let $C$ be an ALC concept that contains only one concept variable $E, T$ be a tree of $C$ built by the algorithm BuildCIT, and $C_{T}$ be a concept represented by the $T$, then $C_{T}$ is logically equivalent to $C$, and is one of the concepts among $\perp, \top, E$, or $\neg E$.

Proof. The concept $C$ must be one of the following four concepts: $\perp, \top, E$, or $\neg E$. If $C=\perp$ or $C=\top$, then the algorithm BuildCIT builds tree $T$, which contains only one node labelling $\perp$ or $\mathrm{T}$. Thus, $C_{T}=\perp$ or $C_{T}=\top$, and $C_{T}$ is logically equivalent to $C$. If $C=E$, then the algorithm BuildCIT builds tree $T$, which contains a root node labelling $\perp$ and the first sub-node labelling $E$. Thus, $C_{T}=\perp \sqcup E=E$, and $C_{T}$ is logically equivalent to $C$. If $C=\neg E$, then the algorithm BuildCIT builds tree $T$, which contains a root node labelling $\perp$ and the second sub-node labelling $\neg E$. Thus, $C_{T}=\perp \sqcup \neg E=\neg E$, and $C_{T}$ is logically equivalent to $C$. Therefore, $C_{T}$ is logically equivalent to $C$, and is one of the concepts among $\perp, \top, E$, or $\neg E$.

Theorem 5. Let $C$ be an ALC concept, $V_{C o n}$ be an atomic concept variable set of $C, V_{R o l}$ be a role concept variable set of $C, T$ be a tree of $C$ built by the algorithm BuildCIT, and $C_{T}$ be a concept represented by the $T$, then $C_{T}$ is logically equivalent to $C$, and each branch of $T$ is an implicate of $C$.
Proof. (1) First the logic equivalence was verified by induction on the number $m$ of concept variables in $C$, let $V=V_{\text {Con }} \cup V_{\text {Rol }}=\left\{E_{1}, \ldots, E_{m}\right\}, E_{k}=A_{k} \in V_{\text {Con }}$ or

$$
E_{l}=Q R_{j} \cdot L_{i} \in V_{R o l}, 1 \leq k<l \leq m, Q \in\{\forall, \exists\} .
$$

$<1>$ Base case: Let $m=1$, according to theorem 4 , then $C_{T}$ is logically equivalent to $C$.

$<2>$ Inductive hypothesis: It was assuming that the theorem was true for all concepts with almost all $\mathrm{m}$ concept variables.

$<3>$ Induction: It was assumed that $C$ had $m+1$ concept variables. Let $E_{\mathrm{i}}$ be any concept variable of $V, E_{i} \in V_{C o n}$ or $E_{i} \in V_{R o l}, 1 \leq i \leq m$, now assuming that $E_{1}$ is an atomic concept variable form $V_{C o n}$, then, it must be proved that:

$$
\begin{aligned}
& C \equiv C_{T}=\left(E_{1} \sqcup C_{\text {BuildCIT }\left(C\left[\perp / E_{1}\right], V_{C o n}-\left\{E_{1}\right\}, V_{R o l}, N_{1}\right)}\right) \\
& \sqcap\left(\neg E_{1} \sqcup C_{\text {BuildCIT }\left(C\left[T / E_{1}\right], Y_{C o n}-\left\{E_{1}\right\}, V_{\text {Rol }}, N_{2}\right)}\right) \\
& \sqcap\left(C_{\text {BuildCIT }\left(C\left[\perp / E_{1}\right], V_{C o n}-\left\{E_{1}\right\}, V_{\text {Rol }}, N_{1}\right) \cap B u i l d C I T\left(C\left[T / E_{1}\right], V_{C o n}-\left\{E_{1}\right\}, V_{\text {Rol }}, N_{2}\right)}\right)
\end{aligned}
$$

According to the inductive hypothesis, we obtain,

$$
\begin{aligned}
& C\left[\perp / E_{1}\right] \equiv C_{\text {BuildCIT }\left(C\left[\perp / E_{1}\right], V_{C o n}-\left\{E_{1}\right\}, V_{R o}, N_{1}\right)}, \\
& C\left[\top / E_{1}\right] \equiv C_{\text {BuildCIT }\left(C\left[T / E_{1}\right], V_{C o n}-\left\{E_{1}\right\}, V_{\text {Rol }}, N_{1}\right)}, \\
& C\left[\perp / E_{1}\right] \sqcup C\left[\top / E_{1}\right] \\
& \equiv C_{\text {BuildCIT } \left.\left(C\left[\perp / E_{1}\right], V_{C o n}-\left\{E_{1}\right\}, V_{R o l}, N_{1}\right)\right) \operatorname{BuildCITT}\left(C\left[T / E_{1}\right], V_{C o n}-\left\{E_{1}\right\}, V_{R o l}, N_{2}\right)} \\
& \text { So, } \\
& C_{T}=\left(E_{1} \sqcup C\left[\perp / E_{1}\right]\right) \sqcap\left(\neg E_{1} \sqcup C\left[\top / E_{1}\right]\right) \\
& \sqcap\left(C\left[\perp / E_{1}\right] \sqcup C\left[\top / E_{1}\right]\right)
\end{aligned}
$$

Let $I=\left\langle\Delta^{I}, \bullet^{I}\right\rangle$ be any model of concept $C$, so $C^{I} \neq \varnothing$, and there exists an individual $a$ such that $a \in C^{I}$. Following are the two cases of the individual $a$.

Case 1, supposing $a \in E_{1}^{I}$, hence, $a \in\left(C\left[\top / E_{1}\right]\right)^{I}$, and $a \in\left(C\left[\perp / E_{1}\right] \sqcup C\left[\top / E_{1}\right]\right)^{I}$. Therefore, $\quad a \in\left(C_{T}\right)^{I}$. Thus, $C^{I} \subseteq\left(C_{T}\right)^{I}$, and $C \sqsubseteq C_{T}$.

Case 2, supposing $a \notin E_{1}^{I}$, hence $\quad a \in\left(\neg E_{1}\right)^{I}$, $a \in\left(C\left[\perp / E_{1}\right]\right)^{I}$, and $a \in\left(C\left[\perp / E_{1}\right] \sqcup C\left[\top / E_{1}\right]\right)^{I}$.therefore, $a \in\left(C_{T}\right)^{I}$. Thus, $C^{I} \subseteq\left(C_{T}\right)^{I}$, and $C \sqsubseteq C_{T}$.

Therefore, $C \sqsubseteq C_{T}$ suggesting that $C_{T} \sqsubseteq C$ is similar. Hence, $C \equiv C_{T}$, that is to say, $C_{T}$ is logically equivalent to $C$.

(2) It was shown that each branch of $T$ is an implicate of $C$. According to the distributive laws of description logic that is similar to the distributive laws of proposition logic, $C_{T}$ is logically equivalent to the concept conjunction of the labels of its branches. Moreover, due to the interpretation of concept conjunction, each branch of $T$ is an implicate of $C$. 
Theorem 6. Let $C$ be the ALC concept, $V_{C o n}$ be an atomic concept variable set of $\mathrm{C}$, and $V_{R o l}$ be a role concept variable set of $\mathrm{C}$. Then algorithm BuildCIT is valid and complete.

Proof. (1) First, the validity of the algorithm was proved. The algorithm BuildCIT built a tree $T$, and according to theorem 5, each branch of $T$ was an implicate of $C$, thereby making $T$ a concept implicate tree of $C$. Thus, the algorithm BuildCIT was proved to be valid.

(2) Now, the complete algorithm is explained below. For any concept $C$, the algorithm BuildCIT can build a corresponding tree $T$, and there does not exist a concept without the corresponding tree. Thus, the algorithm BuildCIT is complete.

\section{TRACTABLE QUERYING}

In this section, for any concept represented by a concept implicate tree, the queries are computable in the linear time depending on the size of the query.

Let $C$ be any ALC concept and $T$ be a concept implicate tree of $C$. There are three queries for ALC concepts, satisfiability-testing, tautology-testing, and subsumption-testing.

Considering the satisfiability-testing, if $T$ contains only one node that is labelled as $\perp$, then $C$ is characterized with unsatisfiability, otherwise with satisfiability. With regard to the tautology-testing, if $T$ contains only one node that is labelled as $\mathrm{T}$, then $C$ has tautology, otherwise $C$ has no tautology. Obviously, these two queries can be addressed in the linear time.

In order to test subsumption between the two concepts, the paper provides some theorems as follows.

Let $c l$ be a clausal concept with a concept literal $L$ or a role concept literal $L$. Then, $c l /\{L\}$ denotes a new clausal concept that deletes the literal $L$ from $\mathrm{cl}$. The prefix of a clausal concept $c l=L_{1} \sqcup L_{2} \sqcup \cdots \sqcup L_{s}$ is a clausal concept of the form $c l^{\prime}=L_{1} \sqcup L_{2} \sqcup \cdots \sqcup L_{t}, 0 \leq t \leq s$. If $t=0$, then the prefix is $\perp$.

Theorem 7. Let $C$ be an ALC concept, and $c l$ be an implicate of $C$ with a literal $L$. Then $(c l /\{L\}) \in \operatorname{Cimp}(C[\perp / L])$.

Proof. Let $I=\left\langle\Delta^{I}, \bullet^{I}\right\rangle$ be any model of concept $C[\perp / L]$, then $(C[\perp / L])^{I} \neq \varnothing$. Now, $I$ is extended to $I^{\prime}=<\Delta^{I^{\prime}}, \bullet^{I^{\prime}}>$ by setting $\Delta^{I}=\Delta^{I^{\prime}}, L^{I^{\prime}}=\varnothing$, then $I^{\prime}$ is a model of concept $C$. Therefore, $(C[\perp / L])^{I}=C^{I^{\prime}}$. Since $c l$ be an implicate of concept $C$, hence, $C^{I^{\prime}} \subseteq c l^{I^{\prime}}$. Moreover, $c l^{I^{\prime}}=(\mathrm{cl} /\{L\})^{I}$. Thus, $(C[\perp / L])^{I} \subseteq(\mathrm{cl} /\{L\})^{I}, \quad$ and $C[\perp / L] \supsetneqq(c l /\{L\})$. Therefore,

$$
(c l /\{L\}) \in \operatorname{Cimp}(C[\perp / L]) .
$$

Theorem 8. Let $C$ be an ALC concept, $V_{C o n}$ be an atomic concept variable set of $C, V_{R o l}$ be a role concept variable set of $C, T$ be a tree of $C$ built by the algorithm BuildCIT, and clausal concept $\mathrm{cl}$ be an implicate of $C$. Then there is a unique prefix of $c l$ that is a branch of $T$.

Proof. It was proved that there is a unique prefix of $\mathrm{cl}$, which is a branch of $T$. By induction on the number $m$ of concept variables in $C$, let $V=V_{\text {Con }} \cup V_{R o l}=\left\{E_{1}, \ldots, E_{m}\right\}$, $E_{k}=A_{k} \in V_{\text {Con }}$ or $E_{l}=Q R_{j} . L_{i} \in V_{R o l}, 1 \leq k<l \leq m$.

1) Base case: Theorem 4 considers the case $m=1$.

2) Inductive hypothesis: It was assumed that the theorem was true for all concepts with almost all $m$ concept variables.

3) Induction: Assuming that $C$ has $m+1$ concept variables. Let

$$
c l=L_{d_{1}} \sqcup L_{d_{2}} \sqcup \cdots \sqcup L_{d_{s}}
$$

be an implicate of $C$, where

$L_{d_{i}}$ is either $E_{d_{i}}$ or $\neg E_{d_{i}}$, and $d_{1}<d_{2}<\cdots<d_{s}$.

Therefore, it must be proved that there is a unique prefix of $c l$ that is a branch of $T$. Let $E_{i}$ be any concept variable of $V, E_{i} \in V_{\text {Con }}$ or $E_{i} \in V_{R o l}, 1 \leq i \leq m$, now assuming that $E_{1}$ is an atomic concept variable of the form $V_{C}$, then it must be proved that there is a unique prefix of $c l$ that is a branch of $C_{T}$, which is the concept

$$
\begin{aligned}
& C_{T}=\left(E_{1} \sqcup C_{\text {BuildCIT }\left(C\left[\perp / E_{1}\right], V_{C o n}-\left\{E_{1}\right\}, V_{\text {Rol }}, N_{1}\right)}\right) \\
& \sqcap\left(\neg E_{1} \sqcup C_{\text {BuildCIT }\left(C\left[T / E_{1}\right], V_{C o n}-\left\{E_{1}\right\}, V_{\text {Rol }}, N_{2}\right)}\right) \\
& \sqcap\left(C_{\text {BuildCIT }\left(C\left[\perp / E_{1}\right], V_{C o n}-\left\{E_{1}\right\}, V_{\text {Rol }}, N_{1}\right) \cap B u i l d C T T\left(C\left[T / E_{1}\right], V_{C o n}-\left\{E_{1}\right\}, V_{\text {Rol }}, N_{2}\right)}\right)
\end{aligned}
$$

By the inductive hypothesis, there is a unique prefix of $\mathrm{cl}$ that is a branch of the intersection of two subtrees $\operatorname{BuildCIT}\left(C\left[\perp / E_{1}\right], V_{C o n}-\left\{E_{1}\right\}, V_{R o l}, N_{1}\right) \quad$ and $\operatorname{BuildCIT}\left(C\left[T / E_{1}\right], V_{C o n}-\left\{E_{1}\right\}, V_{R o l}, N_{2}\right)$. In this case, the theorem is true for the third branch of $C_{T}$. Moreover, if $d_{1}>1$, then nothing is needed to prove, therefore, assuming $d_{1}=1 . L_{l}$ is either $E_{1}$ or $\neg E_{1}$; these are the two cases.

Case 1: Assuming that $L_{1}=E_{1}$, then according to Theorem 7, $\mathrm{cl} /\left\{E_{1}\right\}$ is an implicate of $C\left[\perp / E_{1}\right]$. Moreover, by the inductive hypothesis, there is a unique prefix $G$ of $c l /\left\{E_{1}\right\}$ that is a branch of BuildCTT $\left(C\left[\perp / E_{1}\right], V_{C o n}-\left\{E_{1}\right\}, V_{R o l}, N_{1}\right)$. Therefore, $H=E_{1} \sqcup G$ is a prefix of $c l$ that is a branch of $T$. Now, it is to be proved that $H$ is a unique prefix of $\mathrm{cl}$ by contradiction. Assuming that $H^{\prime}$ is another prefix of $c l$ that is a branch of $T$. Let $H^{\prime}=E_{1} \sqcup G^{\prime}$, then $G^{\prime}$ is a prefix of $c l /\left\{E_{1}\right\}$ that is a branch of BuildCTT $\left(C\left[\perp / E_{1}\right], V_{C o n}-\left\{E_{1}\right\}, V_{R o l}, N_{1}\right)$. However, $G$ is a unique prefix of $c l /\left\{E_{1}\right\}$ by the inductive hypothesis, therefore, $G^{\prime}=G, H^{\prime}=H$. Therefore, $H$ is a unique prefix of $c l$ that is a branch of $T$.

Case 2: Assuming that $L_{1}=\neg E_{1}$, then according to Theorem 7, $\mathrm{cl} /\left\{\neg E_{1}\right\}$ is an implicate of $C\left[\perp / \neg E_{1}\right]$ that is equiva- 
lent to $C\left[\top / E_{1}\right]$. Moreover, by the inductive hypothesis, there is a unique prefix $G$ of $\left.\mathrm{cl} / \neg E_{1}\right\}$ that is a branch of $\operatorname{BuildCTT}\left(C\left[T / E_{1}\right], V_{C o n}-\left\{E_{1}\right\}, V_{R o l}, N_{1}\right)$.

Therefore,

$H=\neg E_{1} \sqcup G$ is a prefix of $c l$ that is a branch of $T$. Now, it is to be proved that $H$ is a unique prefix of $c l$ by contradiction. Assuming that $H^{\prime}$ is another prefix of $c l$ that is a branch of $T$, let $H^{\prime}=\neg E_{1} \sqcup G^{\prime}$, then $G^{\prime}$ is a prefix of $c l\left\{\neg E_{1}\right\}$ that is a branch of BuildCTT(C[T/E $\left.], V_{\text {Con }}-\left\{E_{1}\right\}, V_{R o l}, N_{1}\right)$. However, $G$ is a unique prefix of $\mathrm{cl}\left\{\neg E_{1}\right\}$ by the inductive hypothesis, so $G^{\prime}=G, H^{\prime}=H$. Therefore, $H$ is a unique prefix of $c l$ that is a branch of $T$.

Above all, there is a unique prefix of $c l$ that is a branch of $T$.

Theorem 9. Let $C$ be an ALC concept, $V_{C o n}$ be an atomic concept variable set of $C, V_{R o l}$ be a role concept variable set of $C$, and $T$ be a concept implicate tree of $C$. Then every prime implicate of $C$ is a branch of $T$.

Proof. According to Theorem 8, the prefix of an implicate is unique. Thus, it is obvious that the conclusion holds true.

In example 2, all prime implicates of concept $\mathrm{C}$ are

$$
\begin{aligned}
& A_{1} \sqcup A_{2} \sqcup \forall R_{2} . \neg A_{4}, A_{1} \supsetneqq \forall R_{1} \cdot \exists R_{2} \cdot A_{5}, \\
& \neg A_{2} \sqcup \forall R_{1} \cdot \exists R_{2} \cdot A_{5} .
\end{aligned}
$$

Considering the concept implicate tree $\mathrm{T}$, they both are the branches of $\mathrm{T}$.

Theorem 10. Let $C$ be an ALC concept, $V_{C o n}$ be an atomic concept variable set of $C, V_{R o l}$ be a role concept variable set of $C$, and $T$ be a concept implicate tree of $C$. Then every subsuming implicate (including any prime implicate) of a branch of $\mathrm{T}$ contains the literal, labelling the leaf of that branch.

Proof. According to Theorem 8, it is obvious that this conclusion holds true.

In example 2, an implicate $A_{1} \sqcup A_{2} \sqcup \forall R_{2} . \neg A_{4}$ contains the literal $\forall R_{2} \cdot \neg A_{4}$, which is a label of the leaf of a branch of $\mathrm{T}$. Moreover, all other implicates of $\mathrm{C}$ in $\mathrm{T}$, for example are,

$$
\begin{aligned}
& A_{1} \sqcup A_{2} \sqcup \exists R_{2} \cdot A_{4} \sqcup \forall R_{1} \cdot \exists R_{2} \cdot A_{5}, \\
& A_{1} \sqcup A_{2} \sqcup \forall R_{1} \cdot \exists R_{2} \cdot A_{5}, \\
& A_{1} \sqcup \neg A_{2} \cup \forall R_{2} \cdot \neg A_{4} \sqcup \forall R_{1} \cdot \exists R_{2} \cdot A_{5}, \\
& A_{1} \sqcup \neg A_{2} \sqcup \exists R_{2} \cdot A_{4} \sqcup \forall R_{1} \cdot \exists R_{2} \cdot A_{5}, \\
& A_{1} \sqcup \neg A_{2} \sqcup \forall R_{1} \cdot \exists R_{2} \cdot A_{5}, \\
& A_{1} \sqcup \forall R_{2} \cdot \neg A_{4} \sqcup \forall R_{1} \cdot \exists R_{2} \cdot A_{5}, \\
& A_{1} \sqcup \exists R_{2} \cdot A_{4} \sqcup \forall R_{1} \cdot \exists R_{2} \cdot A_{5},
\end{aligned}
$$

Algorithm Subsume

Input: concept implicate tree $T$ of concept $C$, clausal concept $\mathrm{cl}$; Output: Yes, if $C \sqsubseteq c l$; No, if $C \neq c l$.

1. If $c l=\top$, then return Yes.

2. If tree $T$ has only one node labelled $\perp$, then return Yes.

3. If tree $T$ has only one onde labelled $T$, then return No.

4. If $c l=\perp$, then return No.

5. For each literal $L_{\mathrm{i}}$ of $c l$

If there exists a branch $w$ of $T$, such that $L_{1} \sqcup \cdots \sqcup L_{i}$ is a label of $w$ and $L_{\mathrm{i}}$ is a label of leaf node of $w$, then return Yes.

6. Return No.

Fig. (5). Algorithm Subsume.

$$
\begin{aligned}
& A_{1} \sqcup \forall R_{1} \cdot \exists R_{2} . A_{5}, \\
& \neg A_{1} \sqcup \neg A_{2} \sqcup \forall R_{2} \cdot \neg A_{4} \sqcup \forall R_{1} \cdot \exists R_{2} . A_{5}, \\
& \neg A_{1} \sqcup \neg A_{2} \sqcup \exists R_{2} . A_{4} \sqcup \forall R_{1} \cdot \exists R_{2} . A_{5}, \\
& \neg A_{1} \sqcup \neg A_{2} \sqcup \forall R_{1} \cdot \exists R_{2} \cdot A_{5}, \\
& \neg A_{2} \sqcup \forall R_{2} \cdot \neg A_{4} \sqcup \forall R_{1} \cdot \exists R_{2} . A_{5}, \\
& \neg A_{2} \sqcup \exists R_{2} . A_{4} \sqcup \forall R_{1} \cdot \exists R_{2} . A_{5},
\end{aligned}
$$

and $\neg A_{2} \sqcup \forall R_{1} \cdot \exists R_{2} . A_{5}$, contain the literal $\forall R_{1} \cdot \exists R_{2} \cdot A_{5}$, which is a label of the leaf of a branch of $T$.

Based on the above theorems, if clausal concept $c l=L_{1} \sqcup L_{2} \sqcup \cdots \sqcup L_{d}$ is an implicate of concept $C$, and $T$ is a concept implicate tree of $C$, then there is a unique prefix $c l^{\prime}=L_{1} \sqcup \cdots \sqcup L_{t}$ of $c l$ that is a branch of $T, 1 \leq t \leq d$, and each literal $L_{\mathrm{i}}$ is a label of that branch, $1 \leq i \leq t$, and $L_{\mathrm{t}}$ is a label of the leaf node. Therefore, the study presents the algorithm Subsume as shown in Fig. (5). The main idea is to determine whether $c l$ is an implicate of $C$ if there exists a branch that labelled the prefix of $c l$.

According to the algorithm, it is obvious that the subsumption-testing can be done by traversing a single branch. Therefore, the time complexity is linear depending on the size of the query, but not on the size of $T$. This is an important property of the proposed method.

Theorem 11. Let $C$ be an ALC concept, $T$ be a concept implicate tree of $C$, and $c l$ be a clausal concept. Then it can be decided in the linear time in $|c l|$ whether $C \sqsubseteq c l,|c l|$ denotes the number of all literals in $c l$.

Proof. Considering the algorithm Subsume, it is obvious that the first four steps of the algorithm can be done in linear time. For the fifth step, the algorithm detects all the literals in $\mathrm{cl}$ to decide whether $C \sqsubseteq \mathrm{cl}$. Therefore, determining whether $C \sqsubseteq c l$ can be done in liner time in $|c l|$. 
Above all, three queries for ALC concepts, satisfiabilitytesting, tautology-testing, and subsumption-testing, can be done in linear time depending on the size of the query.

\section{CONCLUSION}

In this paper, knowledge compilation for description logic was presented based on the concept implicate tree. Firstly, the concept implicate tree was defined for the ALC concept. Moreover, the study also provided an algorithm to translate the arbitrary ALC concept into an equivalent concept implicate tree. Finally, it was proved that satisfiability-testing, autology-testing and subsumption-testing were computable in linear time with respect to the concept implicate tree. It was concluded that any query can be done in linear time based on the size of the query, regardless of the size of the concept implicate tree. In other words, the proposed method is an effective method to deal with knowledge compilation for description logic.

\section{CONFLICT OF INTEREST}

The authors confirm that this article content has no conflict of interest.

\section{ACKNOWLEDGEMENTS}

This work was partially supported by Liaoning Province Natural Science Fund Project of China (2015020034), and National Natural Science Foundation of China (61272171), and the Fundamental Research Funds for the Central Universities of China (3132015044).

\section{REFERENCES}

[1] F. Baader, D. Calvanese, and D. McGuinness, The Description Logic Handbook: Theory, Implementation, and Applications. Cambridge University Press, 2003 .

[2] Y. Jiang, Y. Tang, J. Wang, and S. Zhou, "A semantic web oriented description logic", Pattern Recognition and Artificial Intelligence, vol. 20 , pp. 48-54, 2007.

[3] F. Baader, I. Horrocks, and U. Sattler, "Description Logics for the Semantic Web", KI - Künstliche Intelligenz, vol. 16, pp. 57-59, 2002.

[4] F. Baader, I. Horrocks, and U. Sattler, Description Logics. In: S. Staab, and R. Studer, Eds., Handbook on Ontologies, International Handbooks on Information Systems, Springer, 2004, pp. 3-28.

[5] D. Berardi, D. Calvanese, and G. D. Giacomo, "Reasoning on UML class diagrams", Artificial Intelligence, vol. 168, pp. 70-118, 2005.

[6] M. Schmidt-Schauß, and G. Smolka, "Attributive concept descriptions with complements", Artificial Intelligence, vol. 48, pp. 1-26, 1991.
[7] F. M. Donini, M. Lenzerini, D. Nardi, and W. Nutt, "The complexity of concept languges", Information and Computation, vol. 134, pp. $1-58,1997$.

[8] I. Horrocks, and U. Sattler U, "A description logic with transitive and inverse roles and role hierarchies", Journal of Logic and Computation, vol. 9, pp. 385-410, 1999.

[9] I. Horrocks, U. Sattler, and S. Tobies, "Practical reasoning for very expressive description logics", Logic Journal of the IGPL, vol. 8, pp. 239-264, 2000

[10] I. Horrocks, and U. Sattler, "A Tableau Decision Procedure for SHOIQ", In: Proceedings of the $19^{\text {th }}$ International Joint Conference on Artificial Intelligence (IJCAI 2005), Morgan Kaufmann, Los Altos, 2005, pp. 448-453.

[11] I. Horrocks, and U. Sattler, "A tableau decision procedure for SHOIQ", Journal of Automated Reasoning, vol. 39, pp. 249-276, 2007.

[12] I. Horrocks, O. Kutz, and U. Sattler, "The even more irresistible SROIQ", In: Proceedings of the $10^{\text {th }}$ International Conference on Principles of Knowledge Representation and Reasoning (KR 2006), Menlo Park, California: AAAI Press, 2006, pp. 57-67.

[13] A. Darwiche, and P. Marquis, "A knowledge compilation map", Journal of Artificial Intelligence Research, vol. 17, pp. 229-264, 2002.

[14] A. Darwiche, and P. Marquis, "A perspective on knowledge compilation", In: Proceedings of International Joint Conference on Artificial Intelligence (IJCAI), 2001, pp. 175-182.

[15] M. Cadoli, and M. Donini, "A survey on knowledge compilation", AI Communications, vol. 10, pp. 137-150, 1997.

[16] P. Marquis, "Knowledge compilation using theory prime implicates", In: Proceedings of the $14^{\text {th }}$ International Joint Conference on Artificial Intelligence (IJCAI-95), 1995, pp. 837- 843.

[17] A. Darwiche, "Decomposable negation normal form", Journal of the $A C M$, vol. 48, pp. 608-647, 2001.

[18] N. V. Murray, and E. Rosenthal, "Efficient query processing with reduced implicate tries", Journal of Automation Reasoning, vol. 38, pp. 155-172, 2007.

[19] N. V. Murray, and E. Rosenthal, "Linear response time for implicate and implicant queries", Knowledge and Information System, vol. 22, pp. 287-317, 2010.

[20] N. V. Murray, and Erik Rosenthal, "Reduced implicate tries with updates". Journal of Logic and Computation, vol. 20, pp. 261-281, 2010.

[21] B. Selman, and H. Kautz, "Knowledge compilation and theory approximation", Journal of the ACM, vol. 43, pp. 193-224, 1996.

[22] U. Furbach, and C. Obermaier, "Knowledge compilation for description logics", In: Proceedings of the $3^{\text {rd }}$ Workshop on Knowledge Engineering and Software Engineering (KESE), 2007.

[23] U. Furbach, and C. Obermaier, "Precompiling ALC tboxes and query answering", In: Proceedings of the $4^{\text {th }}$ Workshop on Contexts and Ontologies (C\&O-2008) at the $18^{\text {th }}$ European Conference on Artificial Intelligence, Patras, Greece, 2008, pp. 11-15.

[24] U. Furbach, H. Günther, and C. Obermaier, "A knowledge compilation technique for ALC TBoxes", In: Proceedings of the $22^{\text {nd }}$ International Florida Artificial Intelligence Research Society Conference, Sanibel Island, Florida, USA, 2009, pp. 39-44

[25] M. Bienvenu, "Prime implicate normal form for ALC concepts", In: Proceedings of the $23^{\text {rd }}$ National Conference on Artificial Intelligence, 2008, pp. 412-417.

[26] T. Zou, L. Liu, and S. Lv, "Knowledge compilation for description logic based on concept extension rule", Journal of Computational Information Systems, vol. 8, pp. 2409-2416, 2012.

Received: June 10, 2015
(C) Zou and Deng; Licensee Bentham Open.

Revised: July 29, 2015

Accepted: August 15, 2015

This is an open access article licensed under the terms of the (https://creativecommons.org/licenses/by/4.0/legalcode), which permits unrestricted, noncommercial use, distribution and reproduction in any medium, provided the work is properly cited. 